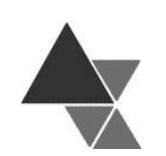

\title{
Segurança Alimentar e Nutricional, Hábitos Alimentares e condições socioeconômicas na Chapada dos Veadeiros no Brasil Central
}

\author{
Fernanda Costa de Aquino ${ }^{1}$, Livia Penna Firme Rodrigues ${ }^{1}$, Estefano Amorim da Silva ${ }^{1}$ e \\ Gabriela Bielefeld Nardoto ${ }^{2}$
}

\begin{abstract}
O objetivo do presente trabalho foi avaliar o grau de insegurança alimentar nos municípios de Cavalcante, Teresina de Goiás e Colinas do Sul, na Chapada dos Veadeiros, Brasil Central. A Segurança Alimentar e Nutricional (SAN) foi avaliada por meio da Escala Brasileira de Insegurança Alimentar (EBIA) e de um roteiro semiestruturado sobre dados socioeconômicos, produção e consumo de alimentos, recordatório alimentar 24 horas e frequência alimentar. Melhor situação (SAN) foi identificada no município de Cavalcante (55\% dos entrevistados), seguido por Teresina e Colinas do Sul (ambos com 45\%). Uma mudança dos hábitos alimentares locais tem sido observada, com a substituição, principalmente no café da manhã e lanche, dos alimentos produzidos localmente, por alimentos processados e industrializados provenientes de mercados. Foram identificadas, entre as populações estudadas, dificuldades de acesso aos alimentos, problemas socioeconômicos, como baixa renda e reduzida escolaridade, que comprometem a SAN nestes municípios.
\end{abstract}

Palavras-chave: Padrões Alimentares; Escala Brasileira de Insegurança Alimentar; Transição Nutricional.

\section{Food and Nutrition Security, Food Habits and Socioeconomic Conditions in Chapada dos Veadeiros in Central Brazil}

The goal of this study was to determine the degree of food insecurity in three municipalities of Central Brazil, Cavalcante, Teresina de Goiás and Colinas do Sul, at Chapada dos Veadeiros. Food and Nutrition Security (FNS) was estimated by using the Brazilian Scale of Food Insecurity (EBIA) and a semi-structured questionnaire about household conditions, food production and consumption, $24 \mathrm{~h}$ food recall method and food frequency. This survey included one adult from 40 domestic units in every municipality. About $55 \%$ of the domestic units in Cavalcante and $45 \%$ in Teresina and Colinas were inserted into FNS program. We found a clear transition from locally produced foods toward to industrialized processed items. Breakfast and coffee breaks were considered the daily meals with the highest percentage of processed items. The survey have shown that the populations from the three municipalities have difficulties related to food access together with socioeconomic issues such as low-income families and lower education, indicating that FNS quite compromised. Therefore, the dependency on food market consumption contributes to an increasing

${ }_{1}^{1}$ Faculdade UnB Planaltina, Universidade de Brasília Campus UnB Planaltina. Área Universitária 1, Vila Nossa Senhora de Fátima, Planaltina, Brasília, DF, CEP: 73345-010, Brasil.

${ }^{2}$ Professora Doutora do Departamento de Ecologia, Instituto de Ciências Biológicas, Campus Universitário Darcy Ribeiro, Universidade de Brasília. Endereço para correspondência: Departamento de Ecologia, Instituto de Ciências Biológicas, Campus Universitário Darcy Ribeiro, Universidade de Brasília, Asa Norte, Brasília, DF, CEP: $70910-900$, Brasil. Telefone: +55 (61) 3107-2985. E-mail: gbnardoto@unb.br 
replacement of locally produced foods to processed foods, which can have a negative impact on the food and nutrition security of these populations.

Keywords: Diet Patterns; Brazilian Food Insecurity Scale; Nutrition Transition.

\section{INTRODUÇÃO}

O Brasil tem vivido muitas transformações nos últimos 50 anos, nos aspectos socioeconômicos, políticos, tecnológicos e também na composição da população, passando de um país rural para urbano. Isso gerou repercussões na vida da população, inclusive na forma de se alimentar, o que afeta diretamente a Segurança Alimentar e Nutricional (SAN). Há décadas atrás a população rural brasileira produzia praticamente todos os alimentos que consumia e a população urbana, se dedicava ao preparo de suas refeições utilizando alimentos sazonais e in natura, comprados nas feiras ou pequenos mercados oriundos do meio rural circunvizinho ${ }^{[1]}$. Já nas últimas décadas, cada vez mais, as populações rurais procuram as capitais e se encaixam, geralmente, entre as famílias de menor poder aquisitivo da zona urbana, aumentando a faixa da população em insegurança alimentar e nutricional [2,3] e as populações urbanas estão cada vez mais expostas a uma imensa variedade de produtos alimentícios, quase sempre transformados industrialmente, e este fenômeno é conhecido como "era dos supermercados" ${ }^{[4,5]}$.

Os alimentos industrializados e produzidos em larga escala em lavouras mecanizadas e vendidos numa ampla rede de grandes, médios e pequenos mercados são os mais utilizados, tanto na cidade como no campo. Alguns fatores foram determinantes para a transformação dos hábitos alimentares nas últimas décadas, como a crescente urbanização, a evolução das formas de distribuição dos alimentos, o papel da mídia para a divulgação nos meios de comunicação, as interações entre as tecnologias de produção e processamento de alimentos, as características físicas de novos alimentos, as respostas dos consumidores a estes alimentos novos e, inclusive, à integração do mercado e o aumento da participação da mulher no mercado de trabalho ${ }^{[6]}$. Com o avanço dos sistemas de produção, ganhou-se em economia de tempo, diminui-se a produção dos alimentos em pequena escala e obteve-se mais praticidade em seu preparo para consumo, além do aumento do prazo de validade $^{[7]}$.

Esta transformação do padrão alimentar, fenômeno que tem ocorrido principalmente no mundo ocidental, é conhecido como transição nutricional ${ }^{[8]}$. A partir da urbanização do meio rural, ocorre a transição nutricional, onde a economia de consumo e a economia de excedentes está dando lugar para a economia de mercado e com isso todas as suas implicações socioculturais ${ }^{[9]}$.

As mudanças ocorridas na alimentação, somadas a outras, devido ao estilo de vida, contribuem para que mais de $60 \%$ da população brasileira (de todas as faixas etárias e de ambos os sexos), seja portadora de Doenças Crônicas Não Transmissíveis (DCNT), um problema nacional, mais grave entre a população de menor renda e de baixa escolaridade. Isso porque os alimentos industrializados e processados contem altos teores de açúcares, gorduras, sal e aditivos químicos, contribuindo fortemente para a incidência dessas doenças na população ${ }^{[1]}$.

Pollan ${ }^{[10]}$ enfatiza que se tornou complicado manter formas tradicionais de alimentação, devido à industrialização dos alimentos. Atualmente, alimentos de consumo tradicional no Brasil, como arroz e feijão, estão sendo substituídos pelos industrializados ${ }^{[11,12,13]}$. De forma geral, a população brasileira não detém conhecimentos relativos à alimentação saudável e raros são os grupos que se beneficiam de programas de educação alimentar e nutricional (EAN) necessária para fazer boas escolhas alimentares; falta conhecimento sobre a origem, produção e qualidade do alimento consumido, priorizando-se quase sempre a praticidade e o baixo custo ${ }^{[13]}$. 
Quando a SAN passou a ser um objetivo público, uma estratégia privilegiada e permanente para se promover o desenvolvimento de um país e quando essa estratégia é colocada sob a ótica do Direito Humano à Alimentação Adequada (DHAA), envolve a soberania alimentar, porque cabe aos povos decidir sobre sua alimentação ${ }^{[14]}$. Portanto, o DHAA se realiza quando todos os cidadãos podem adquirir ou ter acesso a uma alimentação adequada e saudável, ininterruptamente, garantindo não apenas a SAN, mas também a soberania alimentar. Para isso é preciso o desenvolvimento de políticas, programas e ações que coloquem em prática suas obrigações de respeitar, proteger, promover e prover o DHAA, o que inclui, basicamente, a produção, de forma sustentável, e a distribuição de alimentos, o abastecimento e o acesso aos mesmos e a EAN. Uma população tem SAN, quando além de ter acesso à alimentação adequada e saudável, tem garantido também outros direitos, como habitação adequada, acesso a serviços de saúde, educação, dentre outros.

Um dos Indicadores para verificar o nível de SAN da população é a Escala Brasileira de Insegurança Alimentar (EBIA). A aplicação da EBIA nos domićlios ${ }^{[15]}$ apresenta uma vantagem em seu uso, pois mede o fenômeno diretamente a partir da experiência de insegurança alimentar (IA) vivenciada e percebida pelas pessoas afetadas, captando não só a dificuldade de acesso aos alimentos, mas também a dimensão psicossocial da IA. Em dezembro de 2014, o IBGE divulgou o Suplemento da Pesquisa Nacional por Amostra de Domicílio (PNAD) 2013 e seus resultados mostram que, em 2013, a proporção de domicílios com SAN foi estimada em 77,4\%, com Insegurança Alimentar Leve (IAL) 14,8\%, com Insegurança Alimentar Moderada (IAM) 4,6\% e com Insegurança Alimentar Grave (IAG) 3,2\%. Esta última situação atingia 7,2 milhões de pessoas ${ }^{[16]}$, dados que demonstram a vulnerabilidade da população brasileira em relação a SAN. No entanto, estes levantamentos em nível nacional não apresentam informação sobre prováveis especificidades da insegurança alimentar de determinada região ou mesmo município assim como não são capazes de verificar o quanto as vulnerabilidades e circunstâncias regionais podem ser distintas ou similares ao padrão encontrado em nível nacional ${ }^{[17]}$.
A partir dos resultados obtidos por Rodrigues et al. ${ }^{[3]}$, que analisaram algumas dimensões da SAN no município de Alto Paraíso de Goiás, na Chapada dos Veadeiros, região do Brasil Central e verificaram o padrão alimentar e o consumo de alimentos, inclusive os regionais, produzidos e consumidos nos domicílios da população envolvida, decidiu-se estender a pesquisa para outros municípios da região a fim de verificar a extensão desta transição nutricional, com influência sobre a SAN e o potencial de riscos à saúde pública das populações dos municípios da Chapada dos Veadeiros.

Desta forma, o objetivo do presente trabalho foi avaliar o grau de SAN nos municípios de Cavalcante, Teresina e Colinas do Sul, localizados na Chapada dos Veadeiros, Estado de Goiás. A SAN foi avaliada por meio de dados socioeconômicos, utilização da EBIA, levantamento da produção e consumo de alimentos regionais, produzidos e manufaturados e verificação da proporção dos alimentos consumidos, que têm origem local e importada, ou seja, proveniente do processamento e ultra processamento dos alimentos, produzidos fora do local onde foram consumidos.

A partir dos resultados obtidos pretende-se fornecer elementos que possam subsidiar tomadas de decisões acerca de melhores condições de vida para as populações, enfatizando o acesso a uma alimentação saudável, adequada e sustentável, possibilitando, desta forma, fornecer informações relevantes para a formulação de políticas públicas que atendam às necessidades essenciais da população. Entre elas destaca-se a produção e consumo de alimentos, que sejam sustentáveis, do ponto de vista nutricional, de saúde pública e ambiental.

\section{MATERIAL E MÉTODOS}

O presente estudo foi desenvolvido nos municípios de Cavalcante, Teresina e Colinas do Sul, localizados no Estado de Goiás, na região da Chapada dos Veadeiros (Figura 1). Os dados foram coletados na zona urbana, sendo que em Colinas do Sul a coleta foi realizada na zona rural, mais especificamente nos assentamentos Boa Esperança, Angico e Terra Mãe. Segundo o IBGE [18], o município de Cavalcante possui uma população de 9.392 habitantes, com uma 
área total de $6.953,7 \mathrm{~km}^{2}$ e está localizado a aproximadamente $310 \mathrm{~km}$ de Brasília. Teresina abriga uma população de 3.016 habitantes, e sua área é de $774,6 \mathrm{~km}^{2}$, localizado a $315 \mathrm{~km}$ de Brasília. Em Colinas do Sul a população é de 3.523 habitantes, possuindo uma área de $1.708,2 \mathrm{~km}^{2}$ e a uma distância de aproximadamente $293 \mathrm{~km}$ de Brasília.
Ao todo foram entrevistadas 120 pessoas, sendo aplicados 40 questionários, que representam 40 unidades domiciliares, em cada município. Em todos os domicílios foi esclarecido aos entrevistados a finalidade da pesquisa e a solicitação para participar utilizando-se o termo de consentimento global aprovado pelo comitê de ética da ESALQ/USP, sob número de registro COET/053.

Figura 1. Municípios que integram a área compreendida pela Chapada dos Veadeiros - Goiás, Brasil Central

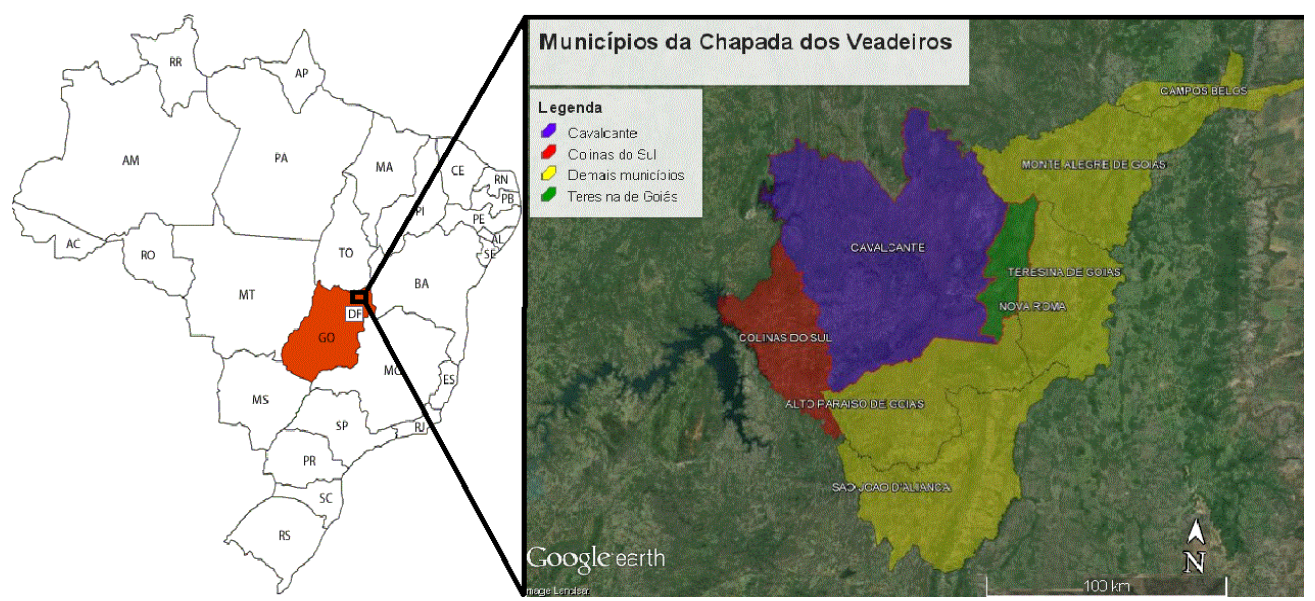

As coletas de dados nos municípios ocorreram nos meses de julho de 2014 e janeiro e outubro de 2015. Os dados foram obtidos por meio da aplicação de um questionário semiestruturado e entrevista com um morador do domicílio. Foram entrevistadas apenas pessoas maiores de 18 anos, ou seja, um representante adulto de cada domicílio, sendo realizado de forma individual e por conveniência. $\mathrm{O}$ questionário, utilizado em pesquisas anteriores ${ }^{[12,19]}$ é composto primeiramente por itens básicos sobre a localização do município e identificação do morador, seguido por questões referentes à escolaridade, benefícios recebidos, ocupação do morador, meio de transporte, situação da moradia, tipo de banheiro, equipamentos utilizados na cozinha, quantidade de cômodos na casa, aparelhos eletrônicos utilizados, entre outros fatores relativos à dimensão socioeconômica.

Informações sobre a produção de alimentos, criação de animais foram obtidas e o consumo alimentar foi identificado por meio do Método Recordatório Alimentar 24 horas e a frequência de consumo de determinados alimentos como café, doces, arroz, trigo, milho, feijões, tubérculos, legumes, farinha de mandioca, folhas verdes, frutas, laticínios, carne bovina, carne suína, embutidos, frango, ovo de galinha, peixe água doce, peixe marinho e frutos do mar. Em relação à frequência de consumo de alimentos, os entrevistados informam a frequência semanal do consumo destes alimentos $(3 \mathrm{x}$ ou mais por semana, até $2 \mathrm{x}$ por semana, nunca ou raramente). O Método Recordatório Alimentar 24 horas é um mecanismo bastante utilizado para a avaliação do consumo alimentar, no qual o entrevistado é indagado sobre o consumo de alimentos e bebidas nas últimas 24 horas ${ }^{[20]}$.

A EBIA possibilita a classificação das famílias em quatro graus de dificuldade de acesso aos alimentos, de acordo com a sua pontuação de respostas positivas aos itens da escala ${ }^{[21]}$. Os 
moradores que têm alguma preocupação ou incerteza quanto ao acesso de alimentos no futuro estão em Insegurança Alimentar Leve (IAL). Os que fizeram uma redução quantitativa ou mudança de padrões alimentares, entre os adultos, estão em Insegurança Alimentar Moderada (IAM) e quando há redução quantitativa de alimentos e mudança dos padrões alimentares entre as crianças e/ou fome, quando alguém fica o dia inteiro sem comer por falta de dinheiro para comprar alimentos, estão em Insegurança Alimentar Grave (IAG) ${ }^{[16]}$.

A EBIA possui a opção de trabalhar com um esquema de filtro, interrompendo a entrevista quando as famílias não convivem com risco de insegurança alimentar. Em caso das respostas afirmativas o questionário irá avaliar, sobretudo, a qualidade da alimentação da família; em seguida, analisa a falta ou não, em termos quantitativos, indicando a deficiência ou insuficiência de alimento para os adultos da família e a deficiência ou insuficiência para as crianças ${ }^{[15]}$.

\section{RESULTADOS E DISCUSSÃO}

\section{SAN, Renda e Escolaridade}

O município que se encontra em melhor situação de SAN neste estudo foi o de Cavalcante, chegando a 55\% dos entrevistados, seguido por Teresina e Colinas do Sul, ambos com 45\% em SAN, conforme pode ser visto na Tabela 1 .

Tabela 1. Classificação nas categorias da escala EBIA para três municípios da Chapada dos Veadeiros, Brasil Central

\begin{tabular}{cccc}
\hline \multirow{2}{*}{$\begin{array}{c}\text { Classificação } \\
(\%)\end{array}$} & \multicolumn{3}{c}{ Municípios } \\
\cline { 2 - 4 } & Cavalcante & $\begin{array}{c}\text { Teresina de } \\
\text { Goiás }\end{array}$ & $\begin{array}{c}\text { Colinas do } \\
\text { Sul }\end{array}$ \\
\hline SAN & 55,0 & 45,0 & 45,0 \\
IAL & 17,0 & 15,0 & 15,0 \\
IAM & 10,0 & 25,0 & 27,5 \\
IAG & 18,0 & 15,0 & 12,5 \\
\hline
\end{tabular}

A SAN significa que há acesso regular e permanente a alimentos de qualidade, em quantidade suficiente, sem prejudicar as outras necessidades essenciais ${ }^{[15]}$. Não podemos afirmar que os alimentos sejam de qualidade, considerando-se que maior parte dos alimentos consumidos são industrializados, conforme será visto mais à frente. No entanto, observa-se que mais da metade dos entrevistados possivelmente tem condições socioeconômicas que permitem o acesso diário à alimentação, por possuírem emprego fixo (Tabela 2). Isso acontece com menos da metade dos entrevistados de Teresina, o que pode ter influenciado no nível de SAN no município. Em Colinas do Sul, os entrevistados são da região rural, fato que justifica a menor percentagem de emprego fixo; mesmo assim $45 \%$ dos entrevistados estão em SAN.

Tabela 2. Ocupação dos entrevistados nos três municípios estudados na Chapada dos Veadeiros, Brasil Central

\begin{tabular}{cccc}
\hline \multirow{2}{*}{$\begin{array}{c}\text { Ocupação } \\
(\%)\end{array}$} & Cavalcante & $\begin{array}{c}\text { Teresina de } \\
\text { Goiás }\end{array}$ & $\begin{array}{c}\text { Colinas do } \\
\text { Sul }\end{array}$ \\
\cline { 2 - 4 } & & 52,5 & 32,5 \\
\hline Salário fixo & 60,0 & 22,5 & 35,0 \\
Autônomo & 25,0 & 25,0 & 32,5 \\
Nenhuma & 15,0 & \multicolumn{3}{c}{ Con } \\
\hline
\end{tabular}

Com relação à Insegurança Alimentar Leve (IAL), que se refere aqueles que têm alguma preocupação ou incerteza quanto ao acesso de alimentos no futuro ${ }^{[15]}$, foi um aspecto que variou pouco entre os entrevistados dos três municípios. Comparando-se nível de escolaridade e a renda dos entrevistados com a SAN, observa-se que, em Cavalcante, estão em IAL os que se encontram entre os $18 \%$ que não concluíram o ensino médio e os $25 \%$ que não possuem salário fixo/ autônomos. A maioria dos entrevistados em Cavalcante $(34 \%)$ possui o ensino médio completo, seguido de ensino superior completo (29\%). Em Teresina são aqueles que estão entre os $12 \%$ que concluíram o ensino fundamental e entre os $7 \%$ que não completaram o ensino médio, encontrando-se na faixa dos $22,5 \%$ que são autônomos. Em Colinas do Sul os entrevistados que estão em IAL, encontram-se principalmente entre os $7 \%$ que não concluíram o ensino médio, entre os $20 \%$ que concluíram o ensino médio, e entre os $35 \%$ autônomos. Observa-se que a preocupação com a falta de alimento no futuro pode estar relacionada a esses fatores, pois não ter completado o ensino básico ou mesmo não ter ensino superior ou renda fixa, pode influenciar na SAN da população estudada.

A Insegurança Alimentar Moderada (IAM), se refere aos adultos que fizeram uma redução quantitativa ou mudança de padrões alimentares ${ }^{[15]}$ 
por não ter acesso aos alimentos. Observou-se que a IAM foi mais grave em Colinas do Sul, região rural, seguida por Teresina e Cavalcante, com a menor proporção. Segundo dados do IBGE ${ }^{[18]}$, a insegurança alimentar nas áreas rurais é superior à região urbana, como é o caso de Colinas de Sul.

Com relação à Insegurança Alimentar Grave (IAG), situação que envolve redução quantitativa de alimentos e mudança dos padrões alimentares entre as crianças e/ou fome, quando alguém fica o dia inteiro sem comer por falta de dinheiro para comprar alimentos ${ }^{[15]}$, foi mais grave em Cavalcante, seguida por Teresina e Colinas do Sul.

Observou-se que os entrevistados em IAM e IAG do município de Cavalcante se encontram entre os $16 \%$ que não concluíram o ensino fundamental e os $3 \%$ que concluíram o ensino fundamental, com uma parcela entre os $25 \%$ que são autônomos e os $15 \%$ que não possuem nenhuma ocupação. Em Teresina, em relação a IAM e IAG, estão entre os $40 \%$ que não concluíram o ensino fundamental e no grupo composto por aqueles $22,5 \%$ que são autônomos e dos $25 \%$ que não possuem nenhuma ocupação. Em Colinas do Sul predominaram os entrevistados com ensino fundamental incompleto, sendo identificada pequena parcela com ensino fundamental completo e ensino médio incompleto e completo e entre os $35 \%$ e $32,5 \%$ de autônomos e aqueles sem ocupação, respectivamente.

É notória a importância de um melhor nível educacional para o alcance da SAN, pois à medida que o indivíduo tem um aumento de anos de estudos, maiores são as oportunidades de emprego que envolve melhor qualificação e consequentemente maiores rendimentos, contribuindo dessa forma para uma alimentação saudável e aumento da SAN. Os dados aqui apresentados confirmam essa premissa de que quanto maior o nível de escolaridade, menor é a insegurança alimentar ${ }^{[21]}$. Nenhum entrevistado com ensino superior completo se enquadrou nos níveis de IAM e IAG.

A partir das análises feitas após a aplicação da EBIA, pode-se verificar que das 120 famílias entrevistadas, em 48,3\% dos domicílios houve garantia de acesso aos alimentos, sendo 22 famílias de
Cavalcante, 18 famílias de Teresina e 18 famílias de Colinas do Sul (famílias classificadas com SAN). Já em $15,8 \%$ das residências, os moradores passaram em algum momento nos últimos 3 meses por IAL ( 7 famílias em Cavalcante, 6 famílias em Teresina e 6 em Colinas do Sul). Já para 35,8\% dos domicílios, o que corresponde a 11 famílias em Cavalcante, 16 famílias em Teresina, e 16 famílias em Colinas do Sul foram classificados com algum grau de Insegurança Alimentar de moderada a grave passando nos últimos 90 dias por restrições alimentares importantes, alterando hábitos alimentares pela falta de alimentos ou mesmo chegando ao ponto mais severo de restringir a alimentação para os moradores menores de 18 anos.

Com relação aos benefícios recebidos nos municípios, na Tabela 3 é possível observar que 25\% dos entrevistados em Cavalcante recebem transferência de renda por meio do Programa Bolsa Família (BPF) e apenas 10\% recebem aposentadoria. Em Teresina, 30\% recebem os benefícios e $27,5 \%$ são aposentados. Já em Colinas do Sul os resultados mostram que 22,5\% são beneficiários do PBF e 27,5\% dos participantes já são aposentados. Nos três municípios os auxílios governamentais predominantes são o Programa Bolsa Família e o Renda Cidadã. Em Colinas do Sul os dados mostram um menor número de pessoas que recebem os benefícios sociais, embora comunidade estudada seja a mais carente.

Tabela 3. Benefícios recebidos pelos entrevistados nos três municípios estudados na Chapada dos Veadeiros, Brasil Central

\begin{tabular}{cccc}
\hline \multirow{2}{*}{ Benefícios } & \multicolumn{3}{c}{ Municípios } \\
\cline { 2 - 4 } & Cavalcante & $\begin{array}{c}\text { Teresina de } \\
\text { Goiás }\end{array}$ & $\begin{array}{c}\text { Colinas do } \\
\text { Sul }\end{array}$ \\
\hline Bolsa Família & 25,0 & 30,0 & 22,5 \\
Aposentadoria & 10,0 & 27,5 & 27,5 \\
Pensão & 0,0 & 10,0 & 7,5 \\
\hline
\end{tabular}

Parte das pessoas entrevistadas não possui nenhum tipo de trabalho remunerado e várias famílias dependem dos benefícios sociais para suprir as necessidades básicas, semelhante ao resultado encontrado na pesquisa de Reinaldo et al. ${ }^{[12]}$. Segundo Segall-Corrêa e Marin-Leon ${ }^{[22]}$ os dados da PNAD 2004 mostraram que os usuários do programa Bolsa 
Família apresentavam prevalências mais altas (15\%) de IAG, mais que o dobro da média nacional e que, a partir de estudos sobre as mudanças ocorridas no perfil de SAN, devido ao acréscimo de renda oriundo deste programa, foi constatado que a cada 10 reais de acréscimo na renda familiar, a chance de estar em SAN aumentava em 8\%. Isto significa que a cada 60 reais recebidos, o domićlio passava de uma situação de IA para SAN, o que demonstra a importância das políticas públicas de transferência de renda como uma estratégia importante para a manutenção da SAN nas populações mais vulneráveis.

Os resultados apresentados comparados com os dados do IBGE ${ }^{[18]}$ enfatizam a ideia de que populações com uma menor renda consomem mais alimentos que são considerados saudáveis como feijão com arroz, possuindo também a possibilidade de terem uma dieta mais diversificada. No entanto, um dado importante para a SAN das famílias entrevistadas é que a maioria delas mantem pequenos cultivos de alimentos em seus quintais, contribuindo para melhorar a alimentação diária.

\section{Condições de moradia}

A maioria dos entrevistados possui equipamentos de cozinha, principalmente geladeira e o fogão a gás, o que facilita a preparação e conservação dos alimentos.

Em Cavalcante, 97,5\% possuem geladeira e 95\% dispõem de fogão a gás, sendo que ainda 27,5\% fazem uso do fogão de lenha. Em Teresina 100\% possuem geladeira e fogão a gás. Em Colinas do Sul $75 \%$ dos entrevistados possuem geladeira (algumas residências não têm disponibilidade de energia elétrica). Nesse mesmo município foi identificado que $82,5 \%$ possuem fogão a gás e $52,5 \%$ fogão a lenha, indícios de serem grupos.

Em relação aos aparelhos eletrônicos, de maneira geral foi observado que nos três municípios a maior parte dos entrevistados possui televisão e telefone. Quanto ao rádio, o equipamento é presente em praticamente todos os municípios/domicílios, com exceção de Colinas do Sul, onde menos de 30\% dos entrevistados afirmaram ter o aparelho. Esses aparelhos eletrônicos, televisão e rádio, influenciam profundamente o consumo alimentar da população, pois, assim como afirma Bleil ${ }^{[23]}$, a propaganda possui forte interferência neste processo de mudança nos hábitos alimentares.

Sobre o tipo de banheiro encontrado nas residências de Cavalcante, foi observado que a maioria $(71 \%)$ dos participantes dispõe de banheiros no interior de suas casas. Em Teresina, também prevaleceu o tipo de banheiro interno (84\%), estando mais presente nas residências deste município comparado a Cavalcante. Não foi observada nenhuma residência com banheiros interno e externo. As informações observadas em Colinas do Sul demonstram uma menor quantidade de banheiros no interior das residências, mesmo que a frequência $(60 \%)$ ainda seja maior quando comparado com a quantidade de banheiros externos (30\%) e residências que não possuem nenhum tipo de banheiro (10\%). Examinando esses dados é factível deduzir o acesso à água, que é um elemento fundamental, tanto para consumo, preparo de alimentos, higiene pessoal e limpeza das casas. Verificou-se que na maioria das casas nos municípios de Cavalcante e Teresina predomina o tipo de banheiro interno; em Colinas do Sul percebe-se que esses valores diminuem, atestando dessa forma uma menor condição socioeconômica que pode afetar a saúde e a segurança alimentar e nutricional. Diante da realidade encontrada em Colinas do Sul, pode-se constatar que é uma situação que ocorre em virtude das condições precárias da localidade. Os dados do CONSEA ${ }^{[21]}$ expõem que a falta de esgotamento sanitário, além de elevar a incidência de doenças, contribui para o crescimento do problema da insegurança alimentar e nutricional.

Em relação à quantidade de cômodos nas casas de Cavalcante, certificou-se que a maioria dos entrevistados detêm de 02 a 07 cômodos em suas residências (4\%), sendo que $43 \%$ têm entre 05 e 07 cômodos e $31 \%$ têm entre 02 e 04 cômodos. No município de Teresina foi observado que $70 \%$ dos entrevistados dispõem de 05 a 07 cômodos, sendo que 30\% possuem entre 02 e 04 cômodos e 08 e 10 cômodos. Em Colinas do Sul, a maioria das casas possui de 02 a 04 cômodos, ou seja, $47 \%$ dos entrevistados. 
O uso de meios de transporte, além de facilitar a locomoção, ajuda no processo de deslocamento para a compra de alimentos (que em sua maioria não são produzidos pelas famílias produção auto consumo) em locais mais distantes, normalmente em mercados. O meio de transporte mais utilizado em Cavalcante, conforme os participantes afirmaram, é o carro, o que é explicado pela maior renda dos moradores $(47 \%$ dos entrevistados possuem carro, $37 \%$ bicicleta e $16 \%$ motocicletas) decorrente do turismo local que gera empregos. Em Teresina de Goiás o uso da bicicleta é maior chegando a $60 \%$, veículo utilizado tipicamente em locais menos urbanizados. $O$ carro também obteve uma porcentagem significativa, sendo citado principalmente pelos entrevistados que possuem salário fixo. No município de Colinas do Sul o uso do carro (na maioria, antigos) é de 37\%, sendo justificado pela distância entre os assentamentos e a cidade. Em relação a motocicletas, $28 \%$ afirmaram dispor deste meio de transporte. A utilização desse recurso auxilia na circulação até a cidade, para onde muitas famílias recorrem quando não possuem o que necessitam nos assentamentos. Ainda assim, 35\% dos participantes certificaram não dispor de nenhum tipo de transporte.

\section{Produção de alimentos e hábitos alimentares}

A aplicação do questionário juntamente com a entrevista possibilitou identificar também os animais criados pelas famílias. No município de Cavalcante foi observada criação apenas de galinha (7,5\% dos entrevistados criavam esse animal). Teresina apresentou maior porcentagem de criação de animais, onde $30 \%$ confirmaram criar galinha, sendo identificado, também, de codorna e de gado. Já em Colinas do Sul, foi detectada uma maior criação de animais chegando a $37,5 \%$ de famílias que criam galinha, além do gado e porco. A distância da cidade os fazem recorrer a este método, levando em consideração que muitos não obtêm meios de transportes para buscar alimentos em outras localidades. Os resultados são semelhantes aos encontrados por Silva et al. ${ }^{[19]}$, que constataram que a criação de animais nos quilombos Kalunga envolve a bovinocultura, suinocultura e criação de galinhas.

Os entrevistados que criam animais $\mathrm{e}$ produzem outros alimentos relataram que muitas vezes não consomem estes alimentos. Há uma preferência pela venda, sendo o dinheiro utilizado, posteriormente, para compra de produtos em mercados. Esta situação pode também ser observada na pesquisa de Reinaldo et al. ${ }^{[12]}$ que enfatizam a priorização da população por produtos de supermercados, mesmo desconhecendo a origem de tais alimentos.

No que diz respeito às frutas cultivadas, foram consideradas tanto as cultivadas na área urbana quanto na rural, e também as provenientes do extrativismo. Foi constatado que, em Cavalcante, a fruta mais cultivada é a manga, no entanto é realizado por apenas 7,5\% dos entrevistados. Há uma variedade de frutas que foram descritas pelos participantes, porém apresentam uma pequena porcentagem de pessoas que realizam o cultivo. Já em Teresina foi observado um maior cultivo e variedade de frutas, sendo também a manga a mais cultivada (27,5\%), seguida da acerola com $20 \%$ de pessoas que realizam seu cultivo. No município de Colinas do Sul, foi identificado um menor cultivo de frutas, onde a mais cultivada é a banana $(7,5 \%$ dos participantes que realizam seu cultivo). Porém foi identificado uma maior produção de verduras, leite e seus derivados e algumas famílias, produzem arroz e feijão, elementos básicos da refeição do brasileiro. Resultados semelhantes foram encontrados por Silva et al. ${ }^{[19]}$, que concluíram que o consumo foi também considerado reduzido, predominando a produção de banana e manga.

No município de Cavalcante foi observado que em todas as refeições mais de $90 \%$ dos alimentos consumidos foram obtidos em mercados. Já em Teresina foi identificado um consumo maior de alimentos produzidos localmente, sendo normalmente frutas e verduras. $\mathrm{O}$ maior índice de consumo de alimentos produzidos (auto consumo) integra a refeição lanche (17\% dos entrevistados), no entanto o percentual de alimentos industrializados chegou a $90 \%$ como componentes do jantar. Este padrão foi similar ao encontrado por Reinaldo et al. ${ }^{[12]}$ junto a comunidades sertanejas (interior do Estado do Rio Grande do Norte) e por Silva et al. ${ }^{[24]} \mathrm{em}$ comunidades ribeirinhas na Amazônia. No caso de Colinas a obtenção de alimentos proveniente dos mercados superou $90 \%$. 
Por meio do método Recordatório Alimentar 24hs. Foi possível observar que nos três municípios o consumo do café, trigo e laticínios predominou (Figura 2). Isso evidencia que a ingestão no café da manhã é restrita, muitas das vezes se limitando ao café com açúcar que contribui com uma proporção importante para o consumo energético diário ${ }^{[25]}$.

Figura 2. Recordatório Alimentar 24 horas do café da manhã para os entrevistados dos três municípios estudados na Chapada dos Veadeiros, GO

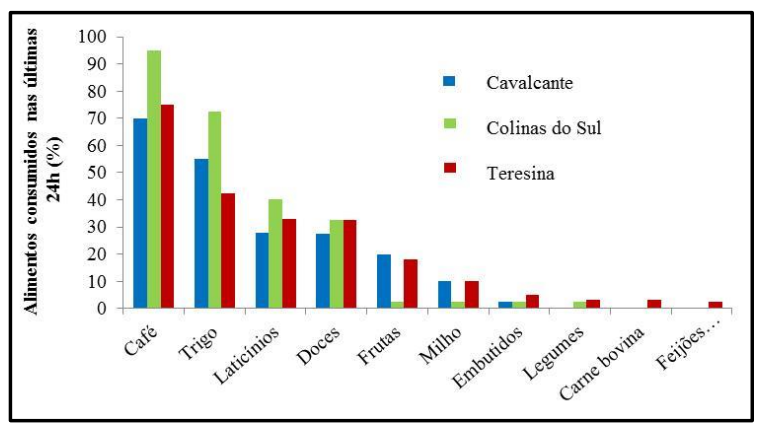

No almoço a base da alimentação nos três municípios é o arroz com feijão, porém estes alimentos não são produzidos pelos entrevistados (Figura 3). Confirma-se, portanto, uma maior dependência com relação aos produtos encontrados nos supermercados, principalmente no que diz respeito ao consumo do arroz (mais de $85 \%$ dos participantes relataram o seu consumo). Outros alimentos que foram bastante citados pelos entrevistados foram os legumes, o frango e a carne bovina.

Figura 3. Resultados obtidos (Recordatório Alimentar 24 horas) para o almoço dos entrevistados de três municípios da Chapada dos Veadeiros - GO

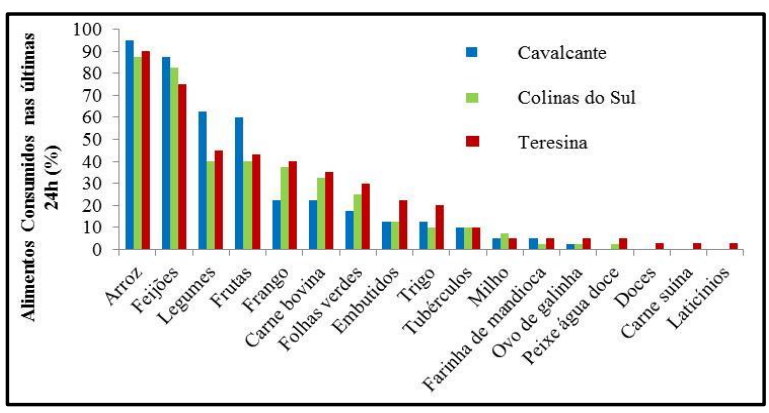

Muitas famílias citaram ingerir (no jantar) os mesmos alimentos preparados para o almoço (para a maioria apenas um mesmo tipo de comida). Assim como os alimentos presentes no almoço se repetem no jantar, há a predominância do arroz, feijão, legumes, carne bovina, frango e frutas, sendo que a fruta mais encontrada em ambas refeições é o tomate (Figura 4).

Figura 4. Resultados obtidos (Recordatório Alimentar 24 horas) para o jantar dos entrevistados de três municípios da Chapada dos Veadeiros - GO

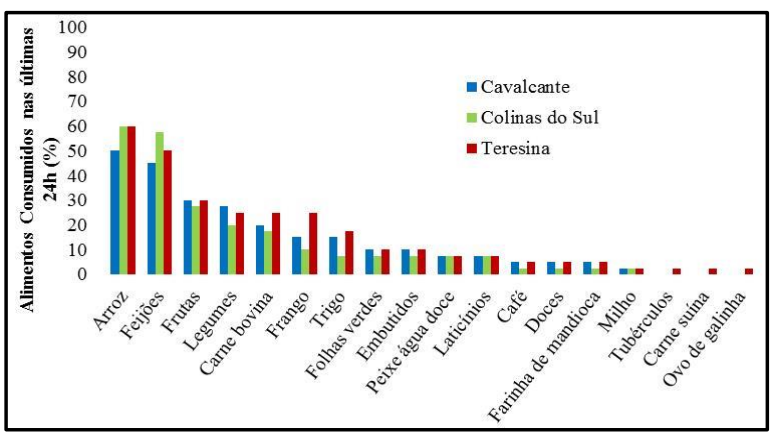

No lanche existem casos, em Teresina e Colinas do Sul, de consumo de alimentos como arroz, feijão e carne bovina, devido à falta de outros tipos de alimentos, sendo o lanche, em algumas famílias, a sua última refeição do dia. Percebe-se um padrão muito semelhante de alimentação entre a população dessas duas localidades. De maneira geral, frutas, trigo e laticínios são os alimentos mais consumidos no lanche nos três municípios (Figura 5). A maioria das frutas citadas pelos entrevistados de Cavalcante são aquelas produzidas localmente e no caso de Teresina e Colinas do Sul uma parcela foi adquirida em supermercados.

Figura 5. Resultados obtidos (Recordatório Alimentar 24 horas) para o lanche dos entrevistados de três municípios da Chapada dos Veadeiros - GO

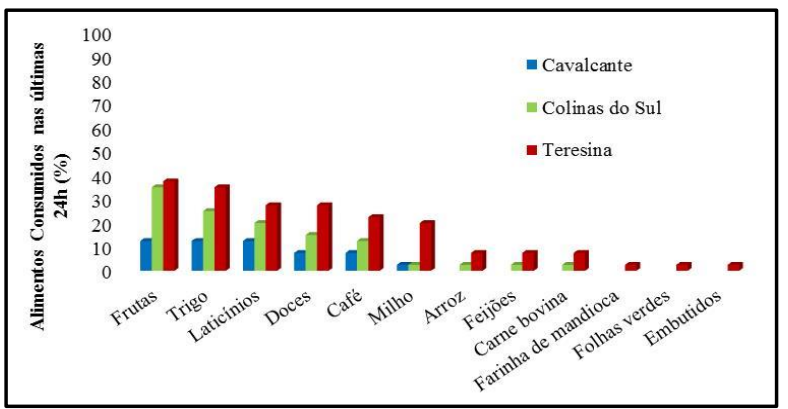


Em relação à frequência de consumo alimentar foi possível constatar que os alimentos mais consumidos ( 3 vezes ou mais na semana) são o arroz, feijão e café, concordando assim com os dados relatados pelos entrevistados no Recordatório Alimentar 24 horas. Esses resultados em relação a frequência alimentar, foram semelhantes ao encontrado na pesquisa realizada por Silva et al. ${ }^{[19]}$, no qual os itens mais consumidos também foram $\mathrm{o}$ arroz, feijão e café. Neste contexto, assim como em outros estudos recentes sobre transição nutricional em diferentes regiões do Brasil [3,12,19,24], os dados apresentados apontam para uma maior dependência do consumo de alimentos processados e/ou industrializados, enfatizando o problema da transição nutricional que gera novos hábitos alimentares, sobretudo em locais onde as condições de vida são regidas por tradições e hábitos locais. Esse maior investimento em produtos processados, os quais em geral são altamente calóricos (com sabores acentuados e atrativos no tocante ao açúcar e gordura) e normalmente de preparo mais fácil e rápido, acaba levando ao favorecimento do seu consumo ${ }^{[26]}$ mas também ao potencial favorecimento de doenças relacionadas à síndrome metabólica, como diabetes do tipo II e pressão alta ${ }^{[27]}$.

\section{CONCLUSÃO}

Este trabalho mostrou que está ocorrendo uma mudança dos hábitos alimentares locais, com a substituição de alimentos produzidos por aqueles provenientes de mercados (frequentemente já processados e industrializados), principalmente no café da manhã e lanche. Nos municípios estudados há dificuldades de acesso aos alimentos, foram identificados problemas socioeconômicos como baixa renda e reduzida escolaridade, condições que dificultam o acesso à SAN.

A dependência do consumo de alimentos adquiridos nos mercados, associada à pequena produção de determinados alimentos, está contribuindo para uma crescente substituição dos alimentos produzidos localmente por produtos industrializados, o que tem ameaçado a segurança alimentar e nutricional dessas populações. Assim, conforme Burlandy et al. ${ }^{[28]}$, a garantia de condições para que a população consiga maior autonomia decisória e, com isso, fazer escolhas alimentares mais saudáveis impões mudanças importantes tanto no âmbito da produção, do processamento e da comercialização dos alimentos.

\section{REFERÊNCIAS}

[1] Brasil. Ministério do Desenvolvimento Social e Combate à Fome. Ministério do Planejamento, Orçamento e Gestão. Instituto Brasileiro de Geografia e Estatística IBGE. Pesquisa Nacional por Amostra de Domicílios Segurança Alimentar - 2004/2009. Rio de Janeiro; 2010.

[2] Amoroso MCM. Alimentação em um bairro pobre de Manaus, Amazonas. Acta Amazônica. 1981;11(3):1-43.

[3] Rodrigues LPF, Carvalho RC, Maciel A, Otanasio PN, Garavello MEPE, Nardoto GB. Food Insecurity in Urban and Rural Areas in Central Brazil: Transition from Locally Produced Foods to Processed Items. Ecol. Food Nutr. 2016;55(4):365-377.

[4] Popkin BM. Global nutrition dynamics: the world is shifting rapidly toward a diet linked with noncommunicable diseases. Am. J. Clin. Nutr. 2006;84:289298.

[5] Nardoto GB, Silva S, Kendall C, Ehleringer JR, Chesson LA, Ferraz ESB, et al. Documenting Geographical Patterns of Human Diet Through Stable Isotope Analysis of Fingernails. Am. J. Phys. Anthropol. 2006;131:137-146.

[6] Oliveira SP, Thébaud-Mony A. Modelo de consumo agroindustrial: Homogeneização ou Diversificação dos Hábitos Alimentares? Rev. Caderno de Debates. 1996;4:113.

[7] Pons SC. Pontos de partida teórico-metodológicos para o Estudo Sociocultural da Alimentação em um contexto de transformação. In: Canesqui AM, Garcia RWD. Antropologia e Nutrição: um diálogo possível. Rio de Janeiro: Editora Fiocruz; 2005. p. 101-128.

[8] Popkin BM. The Nutrition Transition and Obesity in the Developing World. J. Nutr. 2001;131(3):871S-873S.

[9] Piperata B. Nutritional Status of Ribeirinhos in Brazil and the Nutrition Transition. Am. J. Phys. Anthropol. 2007;133:868-878.

[10] Pollan MO. Dilema do Onívoro: uma história natural de quatro espécies. Rio de Janeiro: Intrínseca; 2007. 
[11] Cartocci CM, Neuberger SB. Produção e industrialização de alimentos. Brasília: Universidade de Brasília; 2008.

[12] Reinaldo EDF, Silva MRF, Nardoto GB, Garavello MEPE. Mudanças de Hábitos Alimentares em comunidades Rurais do semiárido da Região Nordeste do Brasil. Interciência. 2015;40(5):330-336.

[13] Brasil. Ministério da Saúde. Secretaria de Atenção à Saúde. Departamento de Atenção Básica. Guia Alimentar para a População brasileira. 2.ed. Brasília (DF); 2014.

[14] Rocha C, Burlandy L, Magalhães R. (Org). Segurança Alimentar e Nutricional: perspectivas, aprendizados e desafios para as políticas públicas. Rio de Janeiro: Editora Fiocruz; 2013.

[15] Corrêa AMS. Insegurança alimentar medida a partir da percepção das pessoas. Estud. Av. 2007;21(60):143-154.

[16] Instituto Brasileiro de Geografia e Estatística - IBGE. Diretoria de Pesquisas. Coordenação de Trabalho e Rendimento. Pesquisa Suplementar de Segurança Alimentar PNAD 2013. Rio de Janeiro; 2014.

[17] Vianna RPT, Hromi-Fielder AJ, Segall-Correa AM, Perez-Escamilla R. Household food insecurity in small municipalities in Northeastern Brazil: a validation study. Food Security. 2012;4:295-303.

[18] Instituto Brasileiro de Geografia e Estatística - IBGE. Censo Demográfico, Geociências, Geografia, Divisão Regional. Rio de Janeiro; 2010.

[19] Silva RJ, Garavello MEPE, Navas R, Nardoto GB, Mazzi EA, Martinelli LA. Transição agroalimentar em comunidades Tradicionais Rurais: $\mathrm{O}$ caso dos remanescentes de quilombo Kalunga, GO. Segur. Aliment. Nutr. 2015;22(1):591-607.

[20] Fisberg RM, Slater B, Marchioni DML, Martini LA. Inquéritos alimentares: métodos e bases científicos. São Paulo: Manole; 2005.
[21] Brasil. Conselho Nacional de Segurança Alimentar e Nutricional - CONSEA. A Segurança Alimentar e Nutricional e o direito humano à alimentação adequada no Brasil. Indicadores e monitoramento da Constituição de 1988 aos dias atuais. Brasília (DF); 2010.

[22] Segall-Corrêa AM, Leon LM. A Segurança Alimentar no Brasil: Proposição e Usos da Escala Brasileira de Medida da Insegurança Alimentar (EBIA) de 2003 a 2009. Segur. Aliment. Nutr. 2009;16(2):1-19.

[23] Bleil SI. O Padrão Alimentar Ocidental: considerações sobre a mudança de hábitos no Brasil. Rev. Cadernos de Debate. 1998;6:1-25.

[24] Silva RJ, Garavello MEPE, Nardoto GB, Mazzi EA, Martinelli LA. Factors influencing the food transition in riverine communities in the Brazilian Amazon. Environ. Dev. $\quad$ Sustain. 2016;18:1-16. DOI http://dx.doi.org/10.1007/s10668-016-9783-x.

[25] Murrieta RSS. Dialética do sabor: alimentação, ecologia e vida cotidiana em comunidades ribeirinhas da Ilha de Ituqui, Baixo Amazonas, Pará. Rev. Antropol. 2001;44(2):39-88.

[26] Drewnowski A, Darmon N. The economics of obesity: dietary energy density and energy cost. Am. J. Clin. Nutr. 2005; 82(Supl):265-273.

[27] Silva H, Padez C. Body size and obesity patterns in Caboclo populations from Pará, Amazônia, Brazil. Ann. Hum. Biol. 2010;37:217-229.

[28] Burlandy L, Magalhães R, Frozi DS. Políticas públicas de segurança alimentar e nutricional. In: Rocha C, Burlandy L, Magalhães R. Segurança Alimentar e Nutricional: perspectivas, aprendizados e desafios para as políticas públicas. Rio de Janeiro: Editora Fiocruz; 2013. p.89-110. 